\title{
Diabetes and change in bone mineral density at the hip, calcaneus, spine, and radius in older women
}

\section{Ann V. Schwartz ${ }^{1}$, Susan K. Ewing ${ }^{1}$, Anne M. Porzig ${ }^{2}$, Charles E. McCulloch ${ }^{1}$, Helaine E. Resnick ${ }^{3}$, Teresa A. Hillier ${ }^{4}$, Kristine E. Ensrud ${ }^{5}$, Dennis M. Black ${ }^{1}$, Michael C. Nevitt ${ }^{1}$, Steven R. Cummings ${ }^{6}$ and $^{2}$ Deborah E. Sellmeyer ${ }^{7}$}

${ }^{1}$ Department of Epidemiology and Biostatistics, University of California San Francisco, San Francisco, CA, USA

2 Endocrine Division, Department of Medicine, University of California San Francisco, San Francisco, CA, USA

${ }^{3}$ Department of Geriatrics, University of Maryland School of Medicine, Baltimore, MD, USA

${ }^{4}$ Kaiser Permanente Center for Health Research Northwest/Hawaii, Portland, OR, USA

${ }^{5}$ VA Medical Center, University of Minnesota, Minneapolis, MN, USA

${ }^{6}$ California Pacific Medical Center, San Francisco, CA, USA

7 Division of Endocrinology, Johns Hopkins School of Medicine, Baltimore, MD, USA

\section{Edited by:}

Peter Vestergaard, Aarhus University Hospital, Denmark

Reviewed by:

Jennifer Tickner, University of

Western Australia, Australia

Florent Elefteriou, Vanderbilt

University, USA

*Correspondence:

Ann V. Schwartz, Department of Epidemiology and Biostatistics,

University of California San Francisco,

185 Berry Street, Suite 5700, 5th

Floor, San Francisco, CA 94107, USA

e-mail: aschwartz@psg.ucsf.edu
Older women with type 2 diabetes mellitus (DM) have higher bone mineral density (BMD) but also have higher rates of fracture compared to those without DM. Limited evidence suggests that DM may also be associated with more rapid bone loss. To determine if bone loss rates differ by DM status in older women, we analyzed BMD data in the Study of Osteoporotic Fractures (SOF) between 1986 and 1998. SOF participants were women $\geq 65$ years at baseline who were recruited from four regions in the U.S. DM was ascertained by selfreport. BMD was measured with dual-energy $x$-ray absorptiometry (DXA) at baseline and at least one follow-up visit at the hip $(N=6624)$ and calcaneus $(N=6700)$ and, on a subset of women, at the spine $(N=396)$ and distal radius $(N=306)$. Annualized percent change in BMD was compared by DM status, using random effects models. Of 6,867 women with at least one follow-up DXA scan, 409 had DM at baseline. Mean age was 70.8 (SD 4.7) years. Baseline BMD was higher in women with DM at all measured sites. In models adjusted for age and clinic, women with prevalent DM lost bone more rapidly than those without DM at the femoral neck $(-0.96$ vs. $-0.59 \% / y e a r, p<0.001)$, total hip $(-0.98$ vs $-0.70 \% /$ year, $p<0.001)$, calcaneus $(-1.64$ vs. $-1.40 \% /$ year, $p=0.005)$, and spine $(-0.33$ vs. $+0.33 \% /$ year, $p=0.033$ ), but not at the distal radius ( -0.97 vs. $-0.90 \% /$ year, $p=0.91$ ). These findings suggest that despite higher baseline BMD, older women with $\mathrm{DM}$ experience more rapid bone loss than those without DM at the hip, spine, and calcaneus, but not the radius. Higher rates of bone loss may partially explain higher fracture rates in older women with DM.

Keywords: type 2 diabetes mellitus, bone mineral density, women, older adults, longitudinal studies

\section{INTRODUCTION}

Type 2 diabetes mellitus (DM) and osteoporosis are two chronic conditions whose prevalence and associated costs continue to increase, particularly among the elderly. Internationally, over $10 \%$ of adults age 60 years and older have DM; in the U.S. the prevalence of DM in this age group is nearly 30\% (Wild et al., 2004; Cowie et al., 2009). The annual number of hip fractures worldwide was estimated as 1.26 million in 1990, and is projected to approximately double by 2025 (Gullberg et al., 1997). In older adults, considerable overlap in DM and osteoporosis would be expected simply due to the high prevalence of each condition. In addition, DM is associated with increased risk of fracture (Janghorbani et al., 2007; Vestergaard, 2007). Paradoxically, cross-sectional studies have demonstrated that DM is associated with normal or higher bone mineral density (BMD) (Buysschaert et al., 1992; Bauer et al., 1993; Orwoll et al., 1996; Vestergaard, 2007). Thus, for any given BMD $T$-score, the fracture risk in those with DM tends to be higher than the corresponding risk for non-diabetic patients (Schwartz et al., 2011).

Although DM is associated with higher baseline BMD, there is some evidence that people with DM may have more rapid bone loss. This could partially account for higher fracture risk at a given BMD since rapid bone loss contributes to fracture risk independent of baseline BMD (Hillier et al., 2007; Ahmed et al., 2010; Cawthon et al., 2012). However, previous reports on the rate of bone loss in older adults with DM have been inconsistent. While several studies have reported accelerated bone loss at the hip in older women with DM, including in the Study of Osteoporotic Fractures (SOF) (Keegan et al., 2004; Cauley et al., 2009; Khalil et al., 2011), slower bone loss has also been reported at the spine (Khalil et al., 2011) and radius (Krakauer et al., 1995). Our goals in this study were to clarify the effects of diabetes on the rate of bone loss and to gain insight into the seemingly paradoxical and poorly understood relationships among diabetes, BMD, 
and fracture. To achieve these goals, we studied the associations between diabetes and rate of bone loss at several skeletal sites in older women enrolled in the Study of Osteoporotic Fractures (SOF), using longitudinal data from 1986 to 1998.

\section{MATERIALS AND METHODS PARTICIPANTS}

The Study of Osteoporotic Fractures (SOF) is a prospective cohort of 9,704 white women aged $\geq 65$ years. Participants were recruited from the community in four U.S. regions: Portland, Oregon; Minneapolis, Minnesota; Baltimore County, Maryland; and the Mononghela Valley near Pittsburgh, Pennsylvania. Enrollment began in 1986, and the current analyses are based on followup data through 1998 (Cummings et al., 1990). Women were recruited irrespective of BMD and fracture history; those unable to walk without assistance and those with bilateral hip replacements were excluded. All women provided written consent, and SOF was approved by the Institutional Review Board at each site.

\section{ASCERTAINMENT OF DIABETES MELLITUS}

At baseline, participants were asked if a physician had ever told them that they had diabetes or "sugar diabetes." Women who answered "yes" to this question were identified as having prevalent DM. Twenty-five women did not answer this question and were excluded. Using the same survey question, incident DM was defined at years $3.5,6,8$, and 10 (corresponding to SOF clinic visits $3,4,5$, and 6) and via medication inventory at years 6, 8, and 10 . Women who did not report diabetes but who were taking diabetic medications were classified as having DM. Nine women included in these analyses reported thiazolidinedione (TZD) use at year 10 .

\section{MEASUREMENT OF BONE MINERAL DENSITY Calcaneal BMD}

Peripheral BMD was measured at the calcaneus using single photon absorptiometry (Osteoanalyzer; Dove Medical Systems) (Cummings et al., 1990) at the baseline, year 6 and year 8 visits in all women and at the year 10 visit in a subset of participants (Figure 1). Of the 9,679 women with baseline DM data, 6,700 women had $\geq 2$ calcaneal measurements and were included in analyses examining the association between DM and change in calcaneal BMD.

\section{Radial BMD}

Distal and proximal radial BMD were measured by single photon absorptiometry. The distal measurement site was just proximal to the junction of the ulna and radius, and the proximal site was $25 \%$ of the total ulnar length distant from the distal site (Cummings et al., 1990). Radial scans were obtained in all women at baseline and in a small subset of women at year 6 . Of the 9,679 women with baseline DM data, 306 had distal BMD measurements and 290 had proximal BMD measurements at both visits and were included in analyses examining the association between DM and changes in distal and proximal radial BMD (Figure 1).

\section{Hip and spine BMD}

Bone mineral density of the total hip, femoral neck, and total lumbar spine was first measured at year 2 (visit 2) using dual-energy

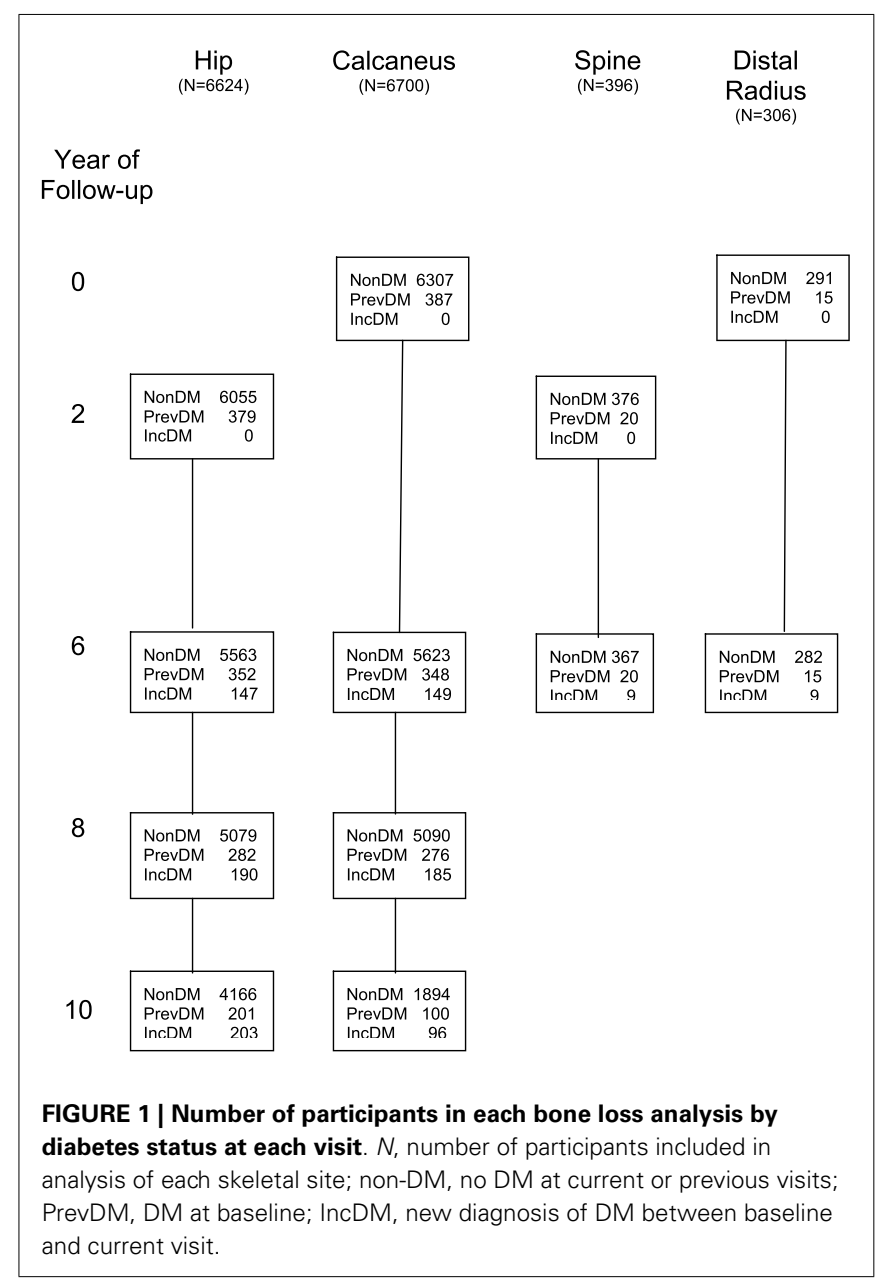

x-ray absorptiometry (DXA) with Hologic QDR-1000 scanners (Hologic, Inc., Bedford, MA, USA). Hip BMD was measured again at years 6 and 8 on the same scanners. At year 10, hip BMD was measured with QDR-1000 scanners for 4,224 women and with QDR-2000 scanners for 346 women. Of the 9,679 women with baseline DM data, 6,624 had $\geq 2$ hip BMD measurements and were included in analyses examining the association between DM and BMD at the total hip and femoral neck (Figure 1). A subset of the 9,679 women had spine BMD measured at year $6(N=479)$, with 396 having spine BMD measurements at both years 2 and 6; these women were included in analyses examining the association between DM and spine BMD.

\section{OTHER MEASUREMENTS}

Weight was measured on a standard balance beam scale, and weight change was calculated by subtracting baseline weight (or year 2 weight for the spine and hip analyses) from current weight. Height was measured by a Harpenden stadiometer (Holtain Ltd., Dyved, UK). Self-reported height at age 25 was collected at baseline; height change was calculated by subtracting height at age 25 from baseline height. Self-reported age at the last menstrual period (LMP) was collected at baseline, and number of years since menopause was calculated by subtracting age at LMP from baseline age (or from year 2 age for hip and spine BMD models). Current use 
of vitamin D and calcium supplements, estrogen preparations, thiazide diuretics, and oral steroids was self-reported at baseline and year 2. Beginning in year 6, participants were asked to bring all prescription and non-prescription medications to the clinic visit for a medication inventory. Self-reported use of calcitonin injections and fluoride pills started at year 2, and self-reported use of etidronate started at year 6. Tobacco use and walking for exercise were self-reported at each visit. Various aspects of physical performance were assessed by trained examiners at each visit. These included grip strength (measured with a handheld Jamar dynamometer using the average of two trials per hand) (Cummings et al., 1995; Seeley et al., 1995), gait speed (measured on a 6-m walking course using the time to complete two trials (Cummings et al., 1995), and ability to rise from a chair five times without using arms (Cummings et al., 1995; Seeley et al., 1995). Peripheral nerve function was assessed at year 2 using esthesiometer testing on the warmed great toe of both feet, using six filaments of increasing size (3.22-6.10, logarithm of force applied, in $0.1 \mathrm{~g}$ ). Women who felt only the 6.10 filament or no filament on either toe were identified as having poor light touch discrimination.

\section{STATISTICAL ANALYSES}

Characteristics of participants were examined according to baseline DM status, using $t$-tests for continuous variables and chisquare tests for categorical variables. Since spine and radial BMD were measured at only two time points, linear regression was used to examine the association between DM and change in BMD at these sites, with results presented as least square means. By contrast, since hip and calcaneal BMD were measured at several time points, random effects models were used to examine the association between DM and change in BMD at these sites. These models account for the between-subject variation and withinsubject correlations among repeated BMD measurements. Time was modeled as a continuous covariate, measured as the number of years between the first BMD and the follow-up BMD scans for each site. Random effects models included the intercept and slope of the BMD measurements over time, thereby allowing for individual time trends for each participant.

All models were adjusted for age and clinic site. The following covariates were initially considered for inclusion in the multivariate models: baseline (or year 2) weight, weight change, baseline (or year 2) height, height loss since age 25 , and current use of any of the following: vitamin D, calcium supplements, estrogen, osteoporosis medications (alendronate, raloxifene, tamoxifen, etidronate, fluoride pills, or calcitonin injections), thiazide, and oral steroids. Also considered for inclusion in the models were current grip strength, walking speed, ability to rise from chair, walking for exercise, years since menopause, current tobacco use, and poor light touch discrimination at year 2 . These covariates were included in the multivariate model if they were significantly associated both with DM in univariate analyses and with change in BMD in the age-clinic-adjusted models at $p$-value $<0.10$. Separate multivariate models were constructed for each skeletal site. Weight loss was added separately to the multivariate models to assess its role as a potential intermediary between DM and change in BMD because it is known to predict bone loss from other studies (Ensrud et al., 2003), and DM is associated with weight loss in the SOF cohort.
Change in BMD is reported as annualized percent change. For hip and calcaneal BMD, the mixed model estimates were used to estimate BMD at each year of follow-up and plotted to visualize changes in BMD over time for each of the DM groups. All analyses were conducted using SAS version 9.1 (SAS Institute Inc., Cary, NC, USA).

\section{RESULTS}

Of the 9,679 women at baseline with known diabetes status, 6,867 had $\geq 2$ BMD measurements during the first 10 years of follow-up. Of these, 409 (6\%) self-reported a physician diagnosis of diabetes at baseline and were categorized as having prevalent DM. Of the remaining 6,458 women who were not diabetic at baseline, 399 (6\%) developed incident DM during follow-up. Characteristics of the 6,867 women included in one or more of these analyses are presented in Table 1. Compared to non-diabetic women, women with prevalent DM had higher baseline BMD at all six sites. Women with prevalent DM had lower grip strength, slower walking speed, and were less likely to walk for exercise and to report estrogen use. Use of alendronate or raloxifene was similar in women with and without DM.

\section{DIABETES AND CHANGE IN HIP BMD}

Among the 6,624 women with $\geq 2$ hip BMD measurements between years 2 and 10,391 and 303 had prevalent and incident $\mathrm{DM}$, respectively. In age and clinic-adjusted models for the femoral neck, women with prevalent DM, incident DM, and those without DM lost an average of $0.96 \%, 0.90$, and 0.59 BMD \%/year, respectively (Table 2 ). At the total hip, both prevalent and incident DM lost $0.98 \%$ /year, while non-DM women lost an average of $0.70 \%$ /year. Although bone loss was more rapid in women with prevalent DM, average BMD remained higher compared with non-DM women throughout 8 years of follow-up (Figure 2).

In multivariate models, mean BMD loss remained significantly greater for the women with prevalent DM compared to women without DM at both the femoral neck $(-0.86$ vs. $-0.54 \%$ /year, $p<0.001)$ and total hip ( -0.86 vs. $-0.59 \% /$ year, $p<0.001)$. Additional adjustment for concurrent weight change slightly attenuated, but did not eliminate, the association between prevalent DM and accelerated BMD loss (Table 2). For incident DM compared to women without DM, multivariate adjustment attenuated the associations for femoral neck $(-0.79$ vs. $-0.54 \% /$ year, $p=0.06)$ and total hip $(-0.77$ vs. $-0.59 \%, p=0.06) \mathrm{BMD}$, and they were no longer statistically significant (Table 2). Further adjustment for concurrent weight change resulted in additional attenuation of these relationships comparing women with incident DM to those without DM.

\section{DIABETES AND CHANGE IN CALCANEAL BMD}

Of the 6,700 women with $\geq 2$ calcaneal BMD measurements between baseline and year 10, 387 and 306 had prevalent and incident DM, respectively. Adjusted for age and clinic, women with prevalent DM lost an average of $1.6 \%$ /year, while those with incident DM and non-DM women lost $1.4 \%$ year (Table 2). Calcaneal BMD was highest among women with prevalent DM, but declined more rapidly over time. Although bone loss was more rapid in those with prevalent $\mathrm{DM}$, average BMD remained higher 
Table 1 | Characteristics ${ }^{a}$ of women by diabetes status.

\begin{tabular}{|c|c|c|c|}
\hline & $\begin{array}{l}\text { Non-DM } \\
(N=6458)\end{array}$ & $\begin{array}{l}\text { Prevalent DM } \\
(N=409)\end{array}$ & $p$-Value \\
\hline Age (years) & $70.7 \pm 4.7$ & $71.0 \pm 4.6$ & 0.35 \\
\hline Weight (kg) & $67.1 \pm 12.0$ & $72.6 \pm 14.8$ & $<0.001$ \\
\hline $\begin{array}{l}\text { Change in weight (V6-BL) } \\
\text { (\%/year) }\end{array}$ & $-0.25 \pm 0.96$ & $-0.41 \pm 0.99$ & 0.012 \\
\hline Height (cm) & $159.6 \pm 5.9$ & $158.9 \pm 6.1$ & 0.017 \\
\hline Height loss since age $25(\mathrm{~cm})$ & $3.1 \pm 2.8$ & $2.9 \pm 2.6$ & 0.095 \\
\hline $\begin{array}{l}\text { Years since menopause } \\
\text { (years) }\end{array}$ & $23.6 \pm 7.8$ & $24.7 \pm 8.1$ & 0.006 \\
\hline Current smoker & $573(8.9)$ & $34(8.3)$ & 0.69 \\
\hline Current vitamin D use & $2958(46.6)$ & 149 (36.9) & $<0.001$ \\
\hline Current calcium use & $2871(44.6)$ & $134(32.8)$ & $<0.001$ \\
\hline Current estrogen use & $1226(19.5)$ & $42(10.4)$ & $<0.001$ \\
\hline $\begin{array}{l}\text { Alendronate taken in last } \\
2 \text { years (V6) }\end{array}$ & 479 (8.9) & $18(6.6)$ & 0.18 \\
\hline Current raloxifene use (V6) & $8(0.2)$ & $1(0.4)$ & 0.36 \\
\hline Current thiazide use & $1513(23.7)$ & $153(37.8)$ & $<0.001$ \\
\hline Current statin use (V4) & $248(3.9)$ & $22(5.5)$ & 0.12 \\
\hline Current oral steroid use & $110(1.7)$ & $4(1.0)$ & 0.27 \\
\hline Grip strength (kg) & $21.3 \pm 4.1$ & $20.8 \pm 4.4$ & 0.020 \\
\hline Walking speed (m/s) & $1.05 \pm 0.20$ & $0.96 \pm 0.21$ & $<0.001$ \\
\hline Inability to rise from chair & $142(2.2)$ & $14(3.4)$ & 0.10 \\
\hline Walk for exercise & 3478 (53.9) & $189(46.2)$ & 0.003 \\
\hline $\begin{array}{l}\text { Poor light touch discrimination } \\
\text { (V2) } \\
\text { Baseline BMD }\end{array}$ & $240(4.3)$ & 35 (9.9) & $<0.001$ \\
\hline Calcaneus BMD $\left(\mathrm{g} / \mathrm{cm}^{2}\right)$ & $0.41 \pm 0.09$ & $0.44 \pm 0.10$ & $<0.001$ \\
\hline Distal radius BMD $\left(\mathrm{g} / \mathrm{cm}^{2}\right)$ & $0.36 \pm 0.08$ & $0.39 \pm 0.08$ & $<0.001$ \\
\hline Proximal radius $\mathrm{BMD}\left(\mathrm{g} / \mathrm{cm}^{2}\right)$ & $0.64 \pm 0.1$ & $0.67 \pm 0.1$ & $<0.001$ \\
\hline $\begin{array}{l}\text { Femoral neck BMD (V2) } \\
\left(\mathrm{g} / \mathrm{cm}^{2}\right)\end{array}$ & $0.65 \pm 0.11$ & $0.69 \pm 0.12$ & $<0.001$ \\
\hline Total hip BMD (V2) $\left(\mathrm{g} / \mathrm{cm}^{2}\right)$ & $0.76 \pm 0.13$ & $0.81 \pm 0.14$ & $<0.001$ \\
\hline $\begin{array}{l}\text { Total lumbar spine BMD (V2) } \\
\left(\mathrm{g} / \mathrm{cm}^{2}\right)\end{array}$ & $0.86 \pm 0.17$ & $0.9 \pm 0.17$ & $<0.001$ \\
\hline
\end{tabular}

Mean (SD), or $N(\%)$. BMD, bone mineral density; $D M$, diabetes mellitus.

${ }^{a}$ Measurement at baseline visit unless otherwise indicated.

compared with non-DM women even after 10 years of followup (Figure 3). The difference in mean loss between the prevalent DM and non-DM groups was significant in the age- and clinic-adjusted model, of borderline significance in the multivariate model, and not significant in a model adjusting for weight change. There was no difference in BMD loss between women with incident DM and those without DM in any of the calcaneal models.

\section{DIABETES AND CHANGE IN LUMBAR SPINE BMD}

Of the 396 women with lumbar spine BMD measurements at years 2 and 6, 20 had prevalent DM and 9 developed incident DM. Adjusted for age and clinic, women with prevalent DM lost BMD ( $-0.33 \% /$ year $)$, while those without DM gained bone $(0.33 \% /$ year; $p=0.03)$ (Table 3). Numbers were too small to assess incident DM separately. Results were similar with multivariable adjustment, including adjustment for concurrent weight change.

\section{DIABETES AND CHANGE IN RADIAL BMD}

Of the 306 women with distal BMD measurements at baseline and year 6, 15 had prevalent DM at baseline and 9 women developed incident DM between baseline and year 6. After adjustment for age and clinic site, women with prevalent DM lost an average of $0.97 \%$ /year at the distal radius while non-DM women lost $0.90 \% /$ year $(p=0.91)$ (Table 3). Numbers were too small to assess incident DM separately. Multivariable adjustment did not substantially alter these results. Of the 290 women with two proximal BMD measurements, 15 had prevalent DM and 7 developed incident DM. Adjusted for age and clinic site, women with prevalent DM gained an average of $0.74 \%$ /year at the proximal radius while nonDM women lost $0.33 \%$ /year $(p=0.14)$. Multivariate adjustment did not substantially alter these results.

\section{DISCUSSION}

Despite their higher baseline BMD, older women with prevalent DM had more rapid bone loss at the total hip, femoral neck, lumbar spine, and calcaneus, but not at the distal or proximal radius, than their non-diabetic counterparts. These results strengthen the evidence for an association between DM and accelerated bone loss, but also clarify that this relationship is site-specific. Our findings extend a previous report from the SOF cohort that DM is associated with accelerated bone loss at the total hip (Cauley et al., 2009), and are in agreement with previous observations of more rapid bone loss at the hip among postmenopausal women with DM in the Health, Aging, and Body Composition Study (Health ABC) and in the placebo group of the Fracture Intervention Trial (FIT) (Keegan et al., 2004; Schwartz et al., 2005). In FIT, there was also a trend toward a faster rate of bone loss at the spine among diabetic women but the difference was not statistically significant (Keegan et al., 2004). Similarly, the Study of Women's Health Across the Nation (SWAN) found an increased rate of bone loss at the total hip among women with DM in the post menopausal but not perimenopausal time period (Khalil et al., 2011). However, SWAN, in contrast to our findings, reported a slower rate of bone loss at the spine in women with DM. Another study found a slower rate of bone loss at the radius over 12 years in 19 adults with DM (average age 52 years), based on BMD $z$-scores (Krakauer et al., 1995). By comparison, we found no differences in the rate of bone loss at the radius.

Thiazolidinedione use may contribute to more rapid bone loss in those with DM. In randomized controlled trials, TZDs have been shown to increase bone loss at the spine and total hip (Berberoglu et al., 2007; Grey, 2008; Borges et al., 2011). However, our findings are not explained by TZD use. The vast majority of follow-up of the SOF cohort took place before the introduction of troglitazone in 1997 and of rosiglitazone and pioglitazone in 1999. Indeed, only nine participants in these analyses reported TZD use at year 10.

Our findings indicate that more rapid bone loss associated with DM is a feature of the hip, spine, and calcaneal sites, but not the radius. In comparison with the other three sites, the radius has a higher proportion of cortical bone and is a non-weight bearing site. In theory, the lack of effect of DM at the radius could result 
Table 2 | Adjusted mean BMD change at the hip and calcaneus by diabetes status.

\begin{tabular}{|c|c|c|c|c|c|c|c|c|}
\hline Site of BMD Change & \multicolumn{3}{|c|}{ Prevalent DM } & \multicolumn{3}{|c|}{ Incident DM } & \multicolumn{2}{|c|}{ Non-DM } \\
\hline \multicolumn{9}{|l|}{ FEMORAL NECK } \\
\hline Adjusted for age and site & -0.96 & $-1.17,-0.77$ & $<0.001$ & -0.90 & $-1.22,-0.59$ & 0.009 & -0.59 & $-0.66,-0.53$ \\
\hline MV without weight change ${ }^{a}$ & -0.86 & $-1.10,-0.63$ & $<0.001$ & -0.79 & $-1.14,-0.44$ & 0.06 & -0.54 & $-0.62,-0.46$ \\
\hline Adjusted for age and site & -0.98 & $-1.15,-0.82$ & $<0.001$ & -0.98 & $-1.22,-0.75$ & 0.001 & -0.70 & $-0.76,-0.65$ \\
\hline MV without weight change ${ }^{a}$ & -0.86 & $-1.06,-0.68$ & $<0.001$ & -0.77 & $-1.03,-0.52$ & 0.06 & -0.59 & $-0.66,-0.53$ \\
\hline MV with weight change & -0.75 & $-0.92,-0.58$ & $<0.001$ & -0.61 & $-0.86,-0.38$ & 0.51 & -0.55 & $-0.61,-0.50$ \\
\hline \multicolumn{9}{|l|}{ CALCANEUS } \\
\hline Adjusted for age and site & -1.64 & $-2.06,-1.25$ & 0.005 & -1.43 & $-2.64,-0.40$ & 0.94 & -1.40 & $-1.54,-1.27$ \\
\hline
\end{tabular}

" $p$-Value for differential rate of BMD loss for prevalent DM vs. non-DM.

" $p$-Value for differential rate of BMD loss for incident DM vs. non-DM.

${ }^{a}$ Adjusted for baseline age, clinic site, baseline height, baseline weight, height change since 25 years since menopause, current vitamin D use, current calcium use, current estrogen use, current use of osteoporosis medications, current thiazide diuretic use, current grip strength, current walking speed, current inability to rise from chair without use of arms, and decreased light touch.

${ }^{b}$ Adjusted for baseline age, clinic site, baseline weight, baseline height, height change since age 25, years since menopause, current vitamin D use, current estrogen use, current thiazide use, current grip strength, current walking speed, current inability to rise from chair without use of arms, and current walking for exercise.

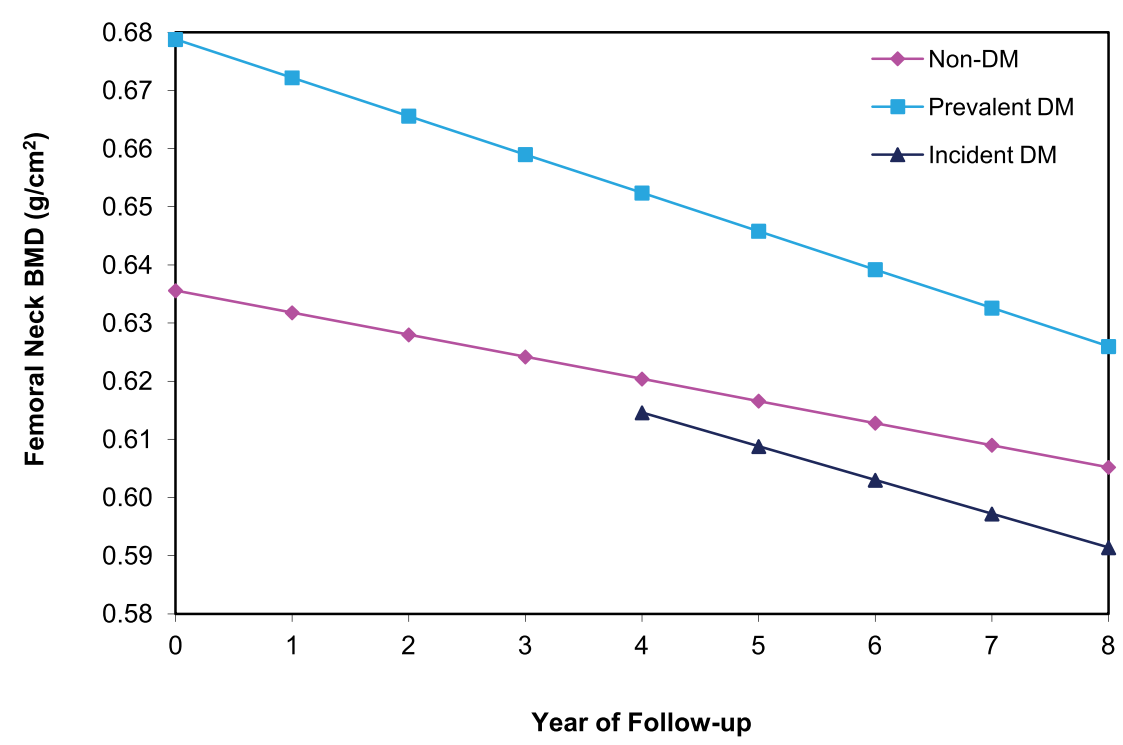

FIGURE 2 | BMD over time at the femoral neck among older women by diabetes status. Mixed model estimates, adjusted for age and clinic site, were used to estimate BMD at each year of follow-up.

from a stronger association with loss of trabecular rather than cortical bone, but cross-sectional studies suggest the opposite (Melton et al., 2008; Petit et al., 2010; Shu et al., 2012; Patsch et al., 2013). To our knowledge, longitudinal studies using quantitative computed tomography (QCT) are not currently available to help disentangle the effect of DM on cortical compared with trabecular bone loss. Another possible explanation is a stronger effect of DM on bone loss in the presence of loading. Other studies indicate that bone geometry, although not bone density, may be negatively affected by DM with a reduction in bone strength relative to load (Petit et al., 2010; Ishii et al., 2012). DM is associated with higher levels of sclerostin (Garcia-Martin et al., 2012), indicating an effect on osteocytes, and with reduced bone formation (Krakauer et al., 1995; Shu et al., 2012). DM may inhibit the ability of osteocytes 


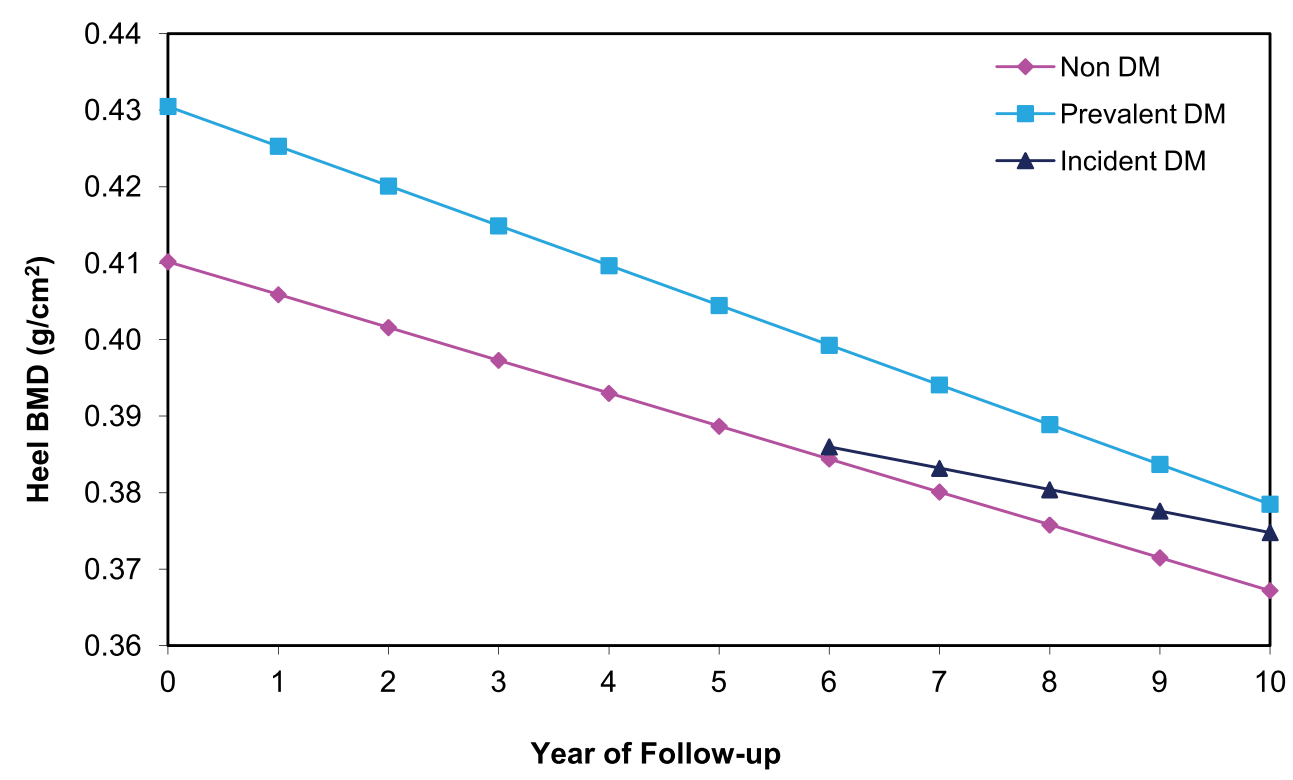

FIGURE 3 | BMD over time at the calcaneus among older women by diabetes status. Mixed model estimates, adjusted for age and clinic site, were used to estimate BMD at each year of follow-up.

Table 3 | Adjusted mean BMD change at the spine and radius by diabetes status.

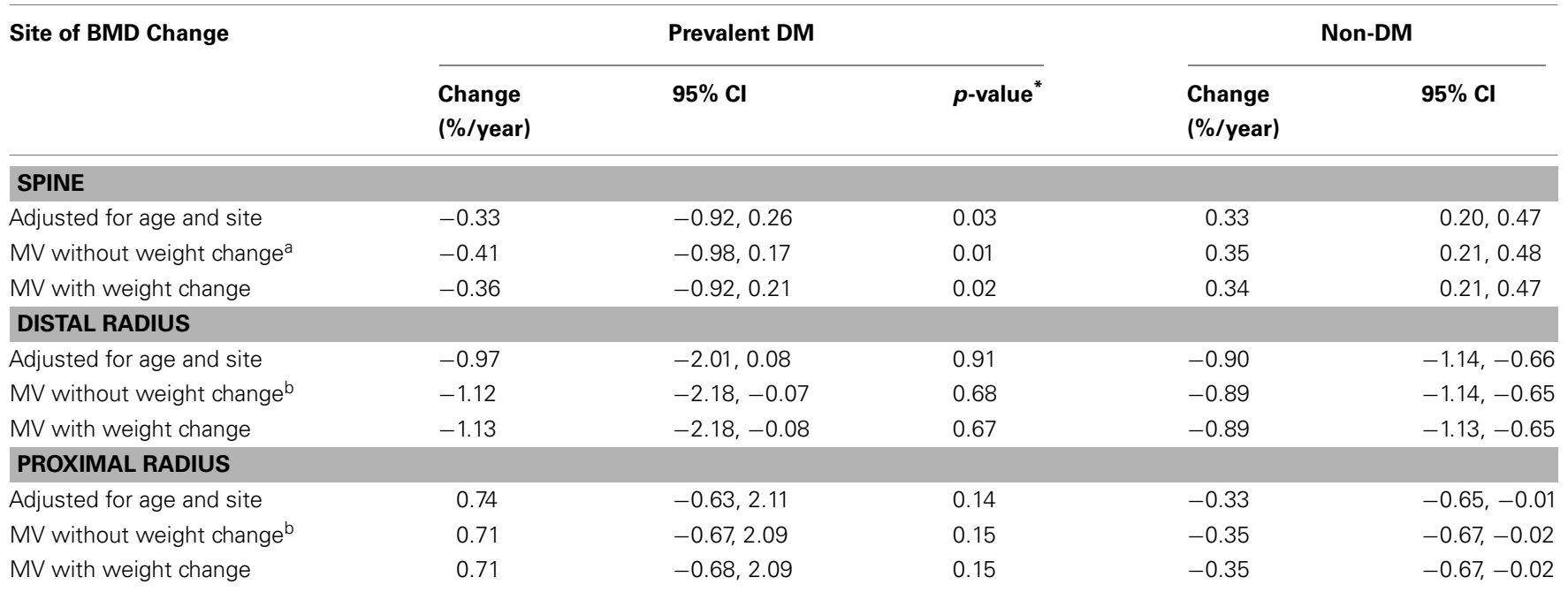

${ }^{*} p$-Value compared to non-DM.

${ }^{a}$ Adjusted for age, clinic site, weight, thiazide diuretic use, and vitamin D use.

${ }^{b}$ Adjusted for age, clinic site, weight, walking for exercise, height change since age 25 , and vitamin D use.

and osteoblasts to respond adequately to load. Thus, at skeletal sites that experience loading, older women with DM may experience greater net loss of bone.

Our results showing higher BMD at baseline are consistent with meta-analyses reporting higher BMD associated with DM (Vestergaard, 2007; Ma et al., 2012). Yet, surprisingly, DM was also associated with more rapid bone loss at the hip, spine, and calcaneus. The rate of bone loss was accelerated among those with incident DM at the hip, but not the calcaneus. Thus, at least for the hip, our results suggest that even at diagnosis, DM has an unfavorable impact on BMD. We also found more rapid weight loss among the diabetic women, despite higher baseline weight. Previous studies have also reported more rapid weight loss with DM (Moritz et al., 1994; Looker et al., 2001; Schwartz et al., 2005). While weight loss is a hallmark of poorly controlled DM, more rapid weight loss was also observed in those with relatively good control (Looker et al., 2001). Weight loss is a strong risk factor for bone loss (Hannan et al., 2000), and more rapid bone loss among 
diabetic women appears to account in part for more rapid bone loss in our study. However, if weight loss were the only mechanism, we would expect our models that included concurrent weight loss to abolish the relationship between bone loss and DM. Instead, although adjustment for concurrent weight change attenuated the effect of accelerated BMD loss among women with prevalent DM, it did not abolish the effect. This indicates that more rapid weight loss does not account for the more rapid bone loss that we observed in women with DM.

Other possible mechanisms for accelerated bone loss with DM include lower levels of insulin-like growth factor 1, changes in calcium homeostasis, increased advanced glycation end products, and decreased blood flow to the lower extremities (Raskin et al., 1978; Vogt et al., 1997; Schwartz et al., 2009). Higher levels of inflammatory cytokines or oxidative stress in those with DM may also drive bone loss (Clowes et al., 2005; Cauley et al., 2007; Manolagas and Almeida, 2007). Reduced exercise in those with DM may increase bone loss (Greendale et al., 1995), but adjustment for physical activity in our models did not account for the observed association between DM and rate of bone loss. Thus, the mechanisms underlying the association between DM and accelerated bone loss merit further exploration.

It has been shown that BMD T-score and FRAX underestimate fracture risk in DM women (Schwartz et al., 2011; Giangregorio et al., 2012). Accelerated bone loss with DM may be a contributing factor as the rate of bone loss predicts fractures independent of baseline BMD (Nguyen et al., 2005; Sornay-Rendu et al., 2005; Bruyere et al., 2007; Hillier et al., 2007; Berger et al., 2009; Ahmed et al., 2010; Cawthon et al., 2012). The reasons for the association between more rapid bone loss and fracture remain controversial. More rapid bone loss may simply be a marker for a lower BMD closer to the time of the fracture. In support of this hypothesis, in the Tromso study the rate of bone loss was no longer associated with fracture risk when models were adjusted for the final BMD measurement, closer to the time of fracture (Ahmed et al., 2010). In contrast, in the Study of Osteoporosis in Men (MrOS) cohort the rate of bone loss predicted hip fracture independent of baseline BMD and final BMD measurements (Cawthon et al., 2012), suggesting that more rapid bone loss may be a marker for changes in

\section{REFERENCES}

Ahmed, L. A., Emaus, N., Berntsen, G. K., Bjornerem, A., Fonnebo, V., Jorgensen, L., et al. (2010). Bone loss and the risk of non-vertebral fractures in women and men: the Tromso study. Osteoporos. Int. 21, 1503-1511. doi:10.1007/s00198009-1102-z

Bauer, D. C., Browner, W. S., Cauley, J. A., Orwoll, E. S., Scott, J. C., Black, D. M., et al. (1993). Factors associated with appendicular bone mass in older women. The Study of Osteoporotic Fractures Research Group. Ann. Intern. Med. 118, 657-665. doi:10.7326/00034819-118-9-199305010-00001

Berberoglu, Z., Gursoy, A., Bayraktar, N., Yazici, A. C., Tutuncu, N.

bone strength that cannot be fully captured by DXA. Fracture in diabetic women is associated with increased cortical porosity, a feature of bone that is not appreciated with DXA scans (Patsch et al., 2013). In our analyses, although DM women had more rapid bone loss, they continued to have higher average hip BMD compared with non-DM women, even after 8 years of follow-up.

Strengths of our study include up to 10 years of follow-up for BMD changes and the ability to adjust for multiple potential confounders, including use of estrogen and osteoporosis medications. A limitation of our study is the relatively small number of women with BMD scans of the spine and radius. Another limitation of this study is the lack of blood tests for ascertainment of DM. It is likely that some participants had undiagnosed diabetes and were incorrectly classified as not having diabetes (Schneider et al., 2012). This misclassification should not have differed by levels of the outcome, changes in BMD. Thus, any misclassification would tend to bias our measures of association between diabetes and change in BMD toward the null. In addition, SOF participants were community dwelling white women, and these results may not apply to other populations. Although analyses were adjusted for multiple factors, the possibility of residual confounding due to factors that were not measured or were measured with error cannot be eliminated.

In conclusion, older women with DM had accelerated bone loss at the hip, spine, and calcaneus, but not at the radius, compared to women without diabetes. Greater concurrent weight loss in the women with DM accounted for some, but not all, of the association between DM and accelerated BMD loss, suggesting that other diabetes-related mechanisms increase bone loss. Despite higher baseline BMD, accelerated bone loss may account, at least in part, for the increased fracture rate observed with DM.

\section{ACKNOWLEDGMENTS}

The Study of Osteoporotic Fractures (SOF) is supported by National Institutes of Health funding. The National Institute on Aging (NIA) provides support under the following grant numbers: R01 AG005407, R01 AR35582, R01 AR35583, R01 AR35584, R01 AG005394, R01 AG027574, and R01 AG027576.

the efficacy and safety of Avandamet (rosiglitazone/metformin) and metformin on long-term glycaemic control and bone mineral density after 80 weeks of treatment in drug-naive type 2 diabetes mellitus patients. Diabetes Obes. Metab. 13, 1036-1046.

Bruyere, O., Roux, C., Detilleux, J., Slosman, D. O., Spector, T. D., Fardellone, P., et al. (2007). Relationship between bone mineral density changes and fracture risk reduction in patients treated with strontium ranelate. J. Clin. Endocrinol. Metab. 92, 3076-3081. doi:10.1210/jc.20062758

Buysschaert, M., Cauwe, F., Jamart, J., Brichant, C., De Coster, P., Magnan, A., et al. (1992). Proximal femur density in type 1 and 2 diabetic patients. Diabete Metab. 18, 32-37.

Cauley, J. A., Danielson, M. E., Boudreau, R. M., Forrest, K. Y., Zmuda, J. M., Pahor, M., et al. (2007). Inflammatory markers and incident fracture risk in older men and women: the Health Aging and Body Composition Study. J. Bone Miner. Res. 22, 1088-1095. doi:10.1359/jbmr.070409

Cauley, J. A., Lui, L. Y., Barnes, D., Ensrud, K. E., Zmuda, J. M., Hillier, T. A., et al. (2009). Successful skeletal aging: a marker of low fracture risk and longevity. The Study of Osteoporotic Fractures (SOF). J. Bone Miner. Res. 24, 134-143. doi:10.1359/jbmr.08 0813 
Cawthon, P. M., Ewing, S. K., Mackey, D. C., Fink, H. A., Cummings, S. R., Ensrud, K. E., et al. (2012). Change in hip bone mineral density and risk of subsequent fractures in older men. J. Bone Miner. Res. 27, 2179-2188. doi:10.1002/jbmr.1671

Clowes, J. A., Riggs, B. L., and Khosla, S. (2005). The role of the immune system in the pathophysiology of osteoporosis. Immunol. Rev. 208, 207-227. doi:10.1111/j.01052896.2005.00334.x

Cowie, C. C., Rust, K. F., Ford, E. S., Eberhardt, M. S., Byrd-Holt, D. D., Li, C., et al. (2009). Full accounting of diabetes and pre-diabetes in the U.S. population in 1988-1994 and 2005-2006. Diabetes Care 32, 287-294. doi:10.2337/dc08-1296

Cummings, S. R., Black, D. M., Nevitt, M. C., Browner, W. S., Cauley, J. A. Genant, H. K., et al. (1990). Appendicular bone density and age predict hip fracture in women. The Study of Osteoporotic Fractures Research Group. JAMA 263, 665-668. doi:10.1001/jama.1990.034400500 59033

Cummings, S. R., Nevitt, M. C., Browner, W. S., Stone, K., Fox, K. M., Ensrud, K. E., et al. (1995). Risk factors for hip fracture in white women. Study of Osteoporotic Fractures Research Group. N. Engl. J. Med. 332, 767-773. doi:10.1056/NEJM1995032333 21202

Ensrud, K. E., Ewing, S. K., Stone, K. L., Cauley, J. A., Bowman, P. J., and Cummings, S. R. (2003). Intentional and unintentional weight loss increase bone loss and hip fracture risk in older women. J. Am. Geriatr. Soc. 51, 1740-1747. doi:10.1046/j.15325415.2003.51558.x

Garcia-Martin, A., Rozas-Moreno, P., Reyes-Garcia, R., Morales-Santana, S., Garcia-Fontana, B., GarciaSalcedo, J. A., et al. (2012). Circulating levels of sclerostin are increased in patients with type 2 diabetes mellitus. J. Clin. Endocrinol. Metab. 97, 234-241. doi:10.1210/jc.2011-2186

Giangregorio, L. M., Leslie, W. D., Lix, L. M., Johansson, H., Oden, A., McCloskey, E., et al. (2012). FRAX underestimates fracture risk in patients with diabetes. $J$. Bone Miner. Res. 27, 301-308. doi:10.1002/jbmr.556

Greendale, G. A., Barrett-Connor, E., Edelstein, S., Ingles, S., and Haile, R. (1995). Lifetime leisure exercise and osteoporosis. The Rancho Bernardo study. Am. J. Epidemiol. 141, 951-959.
Grey, A. (2008). Skeletal consequences of thiazolidinedione therapy. Osteoporos. Int. 19, 129-137. doi:10.1007/s00198-007-0477-y

Gullberg, B., Johnell, O., and Kanis, J. A. (1997). World-wide projections for hip fracture. Osteoporos. Int. 7, 407-413. doi:10.1007/PL00004148

Hannan, M. T., Felson, D. T., DawsonHughes, B., Tucker, K. L., Cupples, L. A., Wilson, P. W., et al. (2000). Risk factors for longitudinal bone loss in elderly men and women: the Framingham Osteoporosis Study. J. Bone Miner. Res. 15, 710-720. doi:10.1359/jbmr.2000.15.4.710

Hillier, T. A., Stone, K. L., Bauer, D. C., Rizzo, J. H., Pedula, K. L., Cauley, J. A., et al. (2007). Evaluating the value of repeat bone mineral density measurement and prediction of fractures in older women: the study of osteoporotic fractures. Arch. Intern. Med. 167, 155-160. doi:10.1001/archinte.167.2.155

Ishii, S., Cauley, J. A., Crandall, C. J., Srikanthan, P., Greendale, G. A., Huang, M. H., et al. (2012). Diabetes and femoral neck strength: findings from the hip strength across the menopausal transition study. J. Clin. Endocrinol. Metab. 97, 190-197. doi:10.1210/jc.2011-1883

Janghorbani, M., Van Dam, R. M., Willett, W. C., and Hu, F. B. (2007). Systematic review of type 1 and type 2 diabetes mellitus and risk of fracture. Am. J. Epidemiol. 166, 495-505. doi:10.1093/aje/kwm106

Keegan, T. H., Schwartz, A. V., Bauer, D. C., Sellmeyer, D. E., and Kelsey, J. L. (2004). Effect of alendronate on bone mineral density and biochemical markers of bone turnover in type 2 diabetic women: the fracture intervention trial. Diabetes Care 27, 1547-1553. doi:10.2337/diacare.27.7.1547

Khalil, N., Sutton-Tyrrell, K., Strotmeyer, E. S., Greendale, G. A., Vuga, M., Selzer, F., et al. (2011). Menopausal bone changes and incident fractures in diabetic women: a cohort study. Osteoporos. Int. 22, 1367-1376. doi:10.1007/s00198010-1357-4

Krakauer, J. C., McKenna, M. J., Buderer, N. F., Rao, D. S., Whitehouse, F. W., and Parfitt, A. M. (1995). Bone loss and bone turnover in diabetes. Diabetes 44, 775-782. doi:10.2337/diabetes.44.7.775

Looker, H. C., Knowler, W. C., and Hanson, R. L. (2001). Changes in BMI and weight before and after the development of type 2 diabetes. Diabetes Care 24, 1917-1922. doi:10.2337/diacare.24.11.1917
Ma, L., Oei, L., Jiang, L., Estrada, K., Chen, H., Wang, Z., et al. (2012). Association between bone mineral density and type 2 diabetes mellitus: a meta-analysis of observational studies. Eur. J. Epidemiol. 27, 319-332. doi:10.1007/s10654012-9674-x

Manolagas, S. C., and Almeida, M. (2007). Gone with the Wnts: \{beta\}catenin, TCF, FOXO, and oxidative stress in age-dependent diseases of bone, lipid, and glucose metabolism. Mol. Endocrinol. 21, 2605-2614 doi:10.1210/me.2007-0259

Melton, L. J. III, Riggs, B. L., Leibson, C. L., Achenbach, S. J., Camp, J. J., Bouxsein, M. L., et al. (2008). A bone structural basis for fracture risk in diabetes. J. Clin. Endocrinol. Metab. 93, 4804-4809. doi:10.1210/jc.20080639

Moritz, D. J., Ostfeld, A. M., Blazer, D., Curb, D., Taylor, J. O., and Wallace, R. B. (1994). The health burden of diabetes for the elderly in four communities. Public Health Rep. 109, 782-790.

Nguyen, T. V., Center, J. R., and Eisman, J. A. (2005). Femoral neck bone loss predicts fracture risk independent of baseline BMD. $J$. Bone Miner. Res. 20, 1195-1201. doi:10.1359/JBMR.050215

Orwoll, E. S., Bauer, D. C., Vogt, T. M., and Fox, K. M. (1996). Axial bone mass in older women. Study of Osteoporotic Fractures Research Group. Ann. Intern. Med. 124, 187-196. doi:10.7326/00034819-124-2-199601150-00001

Patsch, J. M., Burghardt, A. J., Yap, S. P., Baum, T., Schwartz, A. V. Joseph, G. B., et al. (2013). Increased cortical porosity in type 2 diabetic postmenopausal women with fragility fractures. J. Bone Miner. Res. 28, 313-324. doi:10.1002/jbmr. 1763

Petit, M., Paudel, M. L., Taylor, B., Hughes, J., Strotmeyer, E. S., Schwartz, A. V., et al. (2010). Bone mass and strength in older men with type 2 diabetes: the Osteoporotic Fractures in Men Study. J. Bone Miner. Res. 25, 285-291. doi:10.1359/jbmr.090725

Raskin, P., Stevenson, M. R., Barilla, D. E., and Pak, C. Y. (1978). The hypercalciuria of diabetes mellitus: its amelioration with insulin. Clin. Endocrinol. (Oxf.) 9, 329-335. doi:10.1111/j.13652265.1978.tb02218.x

Schneider, A. L., Pankow, J. S., Heiss, G., and Selvin, E. (2012). Validity and reliability of self-reported diabetes in the atherosclerosis risk in communities study. Am. J. Epidemiol. 176, 738-743. doi:10.1093/aje/kws156

Schwartz, A. V., Garnero, P., Hillier, T. A., Sellmeyer, D. E., Strotmeyer, E. S., Feingold, K. R., et al. (2009). Pentosidine and increased fracture risk in older adults with type 2 diabetes. J. Clin. Endocrinol. Metab. 94, 2380-2386. doi:10.1210/jc.20082498

Schwartz, A. V., Sellmeyer, D. E., Strotmeyer, E. S., Tylavsky, F. A., Feingold, K. R., Resnick, H. E., et al. (2005). Diabetes and bone loss at the hip in older black and white adults. J. Bone Miner. Res 20, 596-603. doi:10.1359/JBMR.041 219

Schwartz, A. V., Vittinghoff, E., Bauer, D. C., Hillier, T. A., Strotmeyer E. S., Ensrud, K. E., et al. (2011). Association of BMD and FRAX score with risk of fracture in older adults with type 2 diabetes. JAMA 305, 2184-2192. doi:10.1001/jama.2011.715

Seeley, D. G., Cauley, J. A., Grady, D., Browner, W. S., Nevitt, M C., and Cummings, S. R. (1995). Is postmenopausal estrogen therapy associated with neuromuscular function or falling in elderly women? Study of Osteoporotic Fractures Research Group. Arch. Intern. Med. 155, 293-299. doi:10.1001/archinte.1995.0043003 0087010

Shu, A., Yin, M. T., Stein, E., Cremers, S., Dworakowski, E., Ives, R., et al. (2012). Bone structure and turnover in type 2 diabetes mellitus. Osteoporos. Int. 23, 635-641. doi:10.1007/s00198-0111595-0

Sornay-Rendu, E., Munoz, F., Duboeuf, F., and Delmas, P. D. (2005) Rate of forearm bone loss is associated with an increased risk of fracture independently of bone mass in postmenopausal women: the OFELY study. $J$ Bone Miner. Res. 20, 1929-1935. doi:10.1359/JBMR.050704

Vestergaard, P. (2007). Discrepancies in bone mineral density and fracture risk in patients with type 1 and type 2 diabetes-a meta-analysis. Osteoporos. Int. 18, 427-444. doi:10.1007/s00198-0060253-4

Vogt, M. T., Cauley, J. A., Kuller, L. H., and Nevitt, M. C. (1997). Bone mineral density and blood flow to the lower extremities: the study of osteoporotic fractures. J. Bone Miner. Res. 12, 283-289. doi:10.1359/jbmr.1997.12.2.283 
Wild, S., Roglic, G., Green, A., Sicree, R., and King, H. (2004). Global prevalence of diabetes: estimates for the year 2000 and projections for 2030. Diabetes Care 27, 1047-1053. doi:10.2337/diacare.27.10.2569-a

Conflict of Interest Statement: The authors declare that the research was conducted in the absence of any commercial or financial relationships that could be construed as a potential conflict of interest.

Received: 15 March 2013; accepted: 16 May 2013; published online: 30 May 2013.

Citation: Schwartz AV, Ewing SK, Porzig AM, McCulloch CE, Resnick HE, Hillier TA, Ensrud KE, Black DM, Nevitt MC,
Cummings SR and Sellmeyer DE (2013) Diabetes and change in bone mineral density at the hip, calcaneus, spine, and radius in older women. Front. Endocrinol. 4:62. doi: 10.3389/fendo.2013.00062

This article was submitted to Frontiers in Bone Research, a specialty of Frontiers in Endocrinology.

Copyright (c) 2013 Schwartz, Ewing, Porzig, McCulloch, Resnick, Hillier,
Ensrud, Black, Nevitt, Cummings and Sellmeyer. This is an open-access article distributed under the terms of the Creative Commons Attribution License, which permits use, distribution and reproduction in other forums, provided the original authors and source are credited and subject to any copyright notices concerning any third-party graphics etc. 